VOL. $61(2000) \quad[269-276]$

\title{
SUBDIFFERENTIALS OF CONVEX FUNCTIONS AND SIGMA-CYCLIC MONOTONICITY
}

\begin{abstract}
ARIS DANIILIDIS
The property of $\sigma$-cyclic monotonicity is proposed here to describe subdifferentials of lsc convex functions that are continuous in their domains. It is shown that all monotone operators in $R$ and all densely defined cyclically monotone operators in $R^{n}$ share this property. Examples of a densely defined maximal cyclically monotone operator in a Hilbert space and of a subdifferential of a convex lsc function in $R^{2}$ which are not $\sigma$-cyclically monotone operators are given.
\end{abstract}

\section{INTRODUCTION AND PREREQUISITES.}

In [5], Rockafellar introduced the class of cyclically monotone operators in order to describe the subdifferentials of convex lower semicontinuous (lsc) functions. His main result states that an operator $T: X \rightarrow 2^{X^{*}}$ is included in the subdifferential $\partial f$ of a lsc convex function $f$ if, and only if, it is cyclically monotone. In particular, $T$ coincides with $\partial f$ if, and only if, it is maximal cyclically monotone (with respect to the inclusion of graphs).

In this article we refine the notion of cyclic monotonicity by introducing an infinite variant of it that we call $\sigma$-cyclic monotonicity. We show that this property is always satisfied by the subdifferentials of convex lsc functions that are continuous in their domains, and hence by the subdifferentials of all convex lsc functions in $R$. A simple example shows that subdifferentials of discontinuous convex lsc functions in $R^{2}$ may fail to be $\sigma$-cyclically monotone. However, in finite dimensions, cyclic and $\sigma$-cyclic monotonicity coincide for the class of the densely defined operators. This is no longer true in infinite dimensions. In particular, there exists a convex lsc function $f$ in a separable Hilbert space, for which $\partial f$ is a densely defined maximal cyclically monotone operator, without being $\sigma$-cyclically monotone.

Before we proceed we fix our notation: $X$ will be a Banach space with dual space $X^{*}$. In particular, we denote by $\ell^{2}(N)$ the Hilbert space of square summable sequences,

Received 17th June, 1999

This research was supported by the TMR post-doctoral grant ERBFMBI CT 983381. The author is grateful to D. Aussel, N. Hadjisavvas and J.-P. Penot for having read a preliminary version of the manuscript.

Copyright Clearance Centre, Inc. Serial-fee code: 0004-9727/00 \$A2.00+0.00. 
by $\ell^{1}(N)$ the Banach space of absolutely summable sequences and by $\ell^{\infty}(N)$ the Banach space of bounded sequences. We also denote by $c_{00}(N)$ the space of eventually null sequences. For any $x \in X$ and $x^{*} \in X^{*}$ we denote by $x^{*}(x)$ the value of $x^{*}$ at $x$. For $x \in X$ and $\varepsilon>0$ we denote by $B_{\varepsilon}(x)$ the closed ball centred at $x$ with radius $\varepsilon>0$. Finally for $x, y \in X$ we denote by $[x, y]$ the closed segment $\{t x+(1-t) y: t \in[0,1]\}$. The segments $(x, y],[x, y)$ and $(x, y)$ are defined analogously. Throughout this article we always deal with multivalued operators $T$ defined on $X$ and taking values into $2^{X^{*}}$ (the space of subsets of $X^{*}$ ), and with proper convex lsc functions $f: X \rightarrow R \cup\{+\infty\}$ which are not identically equal to $\{+\infty\}$. We set $\operatorname{dom}(f):=\{x \in X: f(x) \in R\}$ for the domain of $f$ and $\operatorname{dom}(T):=\{x \in X: T(x) \neq \emptyset\}$ for the domain of the multivalued operator $T$.

A function $f: X \rightarrow R \cup\{+\infty\}$ is said to be continuous (respectively lower semicontinuous), if it is continuous (respectively lower semicontinuous) at every point $x \in X$, where $R \cup\{+\infty\}$ is equipped with the topology generated by the family $\Im_{R} \cup\{(x,+\infty], x \in R\}\left(\Im_{R}\right.$ being the usual topology of $\left.R\right)$. Note that such functions may take infinite values, as for instance the function $f: R \rightarrow R \cup\{+\infty\}$ with $f(x)=1 / x$ if $x>0$ and $+\infty$ if $x \leqslant 0$. On the other hand, we say that a function $f$ is continuous on a subset $S$ of its domain, if its restriction to $S$ is a continuous (real-valued) function, see also [4, p.82]. The class of convex lsc functions which are continuous in their domain is much larger than the one of convex continuous functions. In particular, it contains the indicator functions of closed convex sets, as well as many other non-continuous functions, see [3, Example 3.8 (a)].

We recall that the subdifferential $\partial f$ of the function $f$ at the point $x_{0} \in \operatorname{dom}(f)$ is given by the formula below:

$$
\partial f\left(x_{0}\right)=\left\{x^{*} \in X^{*}: f(x)-f\left(x_{0}\right) \geqslant x^{*}\left(x-x_{0}\right), \forall x \in X\right\}
$$

For a lsc convex function $f$, the above subdifferential coincides with the ClarkeRockafellar subdifferential $\partial^{\dagger} f\left(x_{0}\right)$ which was defined in [6] (see also [2]) as follows:

$$
\partial^{\dagger} f\left(x_{0}\right)=\left\{x^{*} \in X: x^{*}(d) \leqslant f^{\dagger}\left(x_{0}, d\right), \forall d \in X\right\} .
$$

where $f^{\dagger}\left(x_{0}, d\right)$ is the generalised derivative of $f$ at $x_{0}$ in the direction $d \in X$ :

$$
f^{\dagger}\left(x_{0}, d\right)=\sup _{\varepsilon>0} \limsup _{\substack{x \rightarrow x_{0} \\ t>0^{+}}} \inf _{d^{\prime} \in B_{\varepsilon}(d)} \frac{f\left(x+t d^{\prime}\right)-f(x)}{t}
$$

where $t \searrow 0^{+}$indicates the fact that $t>0$ and $t \rightarrow 0$, and $x \rightarrow_{f} x_{0}$ means that both $x \rightarrow x_{0}$ and $f(x) \rightarrow f\left(x_{0}\right)$.

\section{Main Results}

Let $T: X \rightarrow 2^{X^{*}}$ be a multivalued operator. We recall from [3] the following definition: 
DEFINITION 1: $T$ is called cyclically monotone, if for any $n \in N$, for every $x_{0}, x_{1}, \ldots, x_{n}$ in $X$ and every $x_{0}^{*} \in T\left(x_{0}\right), x_{1}^{*} \in T\left(x_{1}\right), \ldots, x_{n}^{*} \in T\left(x_{n}\right)$ one has

$$
\sum_{i=0}^{n} x_{i}^{*}\left(x_{i+1}-x_{i}\right) \leqslant 0
$$

where $x_{n+1}:=x_{0}$.

We recall from [5] the following fundamental theorem.

TheOREM 2. $T$ is cyclically monotone if, and only if, $T \subseteq \partial f$ for some convex Isc function $f$.

As shown in [5], if we assume that $T$ is maximal cyclically monotone, then the above inclusion becomes an equality and the function $f$ turns out to be unique.

Let us now introduce the notion of $\sigma$-cyclic monotonicity.

Definition 3: $T$ is called $\sigma$-cyclically monotone, if for every sequence $\left(x_{n}\right)_{n=0}^{+\infty}$ in $X$ such that $\lim _{n \rightarrow \infty} x_{n}=x_{0}$ and for every $x_{i}^{*} \in T\left(x_{i}\right)$ (for $i=0,1,2, \ldots$ ) one has

$$
\limsup _{n \rightarrow \infty} \sum_{i=0}^{n} x_{i}^{*}\left(x_{i+1}-x_{i}\right) \leqslant 0 .
$$

It is easily seen that every $\sigma$-cyclically monotone operator is cyclically monotone. Indeed, given a multivalued operator $T$ and a finite sequence $\left\{x_{0}, x_{1}, \ldots, x_{n}\right\}$ in $\operatorname{dom}(T)$, one can define an infinite sequence $\left(x_{k}\right)_{k=0}^{+\infty}$ in $\operatorname{dom}(T)$, by setting $x_{k}=x_{0}$, for $k \geqslant n+1$. Then relation (5) clearly yields (4).

The following proposition shows that the class of $\sigma$-cyclically monotone operators contains the subdifferentials of functions which are continuous on their domain, thus in particular these of convex continuous functions. (Note also that the proof below does not require the convexity of $f$.)

Proposition 4. Suppose that $f: X \rightarrow R \cup\{+\infty\}$ is continuous on $\operatorname{dom}(\partial f)$ and $T \subseteq \partial f$. Then $T$ is $\sigma$-cyclically monotone.

Proof: Consider any sequence $\left(x_{n}\right)_{n=0}^{+\infty}$ in $\operatorname{dom}(T)$ such that $\lim _{n \rightarrow \infty} x_{n}=x_{0}$. For any $x_{n}^{*} \in T\left(x_{n}\right)$, the inclusion $T\left(x_{n}\right) \subseteq \partial f\left(x_{n}\right)$ together with (1) implies:

$$
f\left(x_{n+1}\right) \geqslant f\left(x_{n}\right)+x_{n}^{*}\left(x_{n+1}-x_{n}\right)
$$

for $n=0,1,2, \ldots$. Adding the above inequalities we get

$$
\sum_{i=0}^{n} x_{i}^{*}\left(x_{i+1}-x_{i}\right) \leqslant f\left(x_{n+1}\right)-f\left(x_{0}\right) .
$$

Taking the limit as $n \rightarrow+\infty$, and using the continuity of $f$ on $\operatorname{dom}(\partial f)$, we conclude that $\limsup _{n \rightarrow \infty} \sum_{i=0}^{n} x_{i}^{*}\left(x_{i+1}-x_{i}\right) \leqslant 0$. 
One deduces that the subdifferentials of indicator functions of convex sets are $\sigma$ cyclically monotone operators. The same is true for the subdifferentials of all lsc convex functions of one variable. More generally we show the following:

Corollary 5. Let $T: R \rightarrow 2^{R}$ be a monotone operator (that is, $T$ satisfies (4) for $n=1$ ). Then $T$ is $\sigma$-cyclically monotone.

ProOF: It is known that every monotone operator in $R$ is cyclically monotone (see [5]), hence Theorem 2 ensures the existence of a lsc convex function $f: R \rightarrow R \cup\{+\infty\}$ such that $T \subseteq \partial f$. We shall show that $f$ is continuous on its domain (which is a segment of $R$ ), hence the result will follow from Proposition 4. Since $f$ is continuous at every interior point of its domain, it suffices to consider only the (eventual) case of a point $x_{0}$ in $\operatorname{dom}(f)$ such that $x_{0} \leqslant x$ for all $x \in \operatorname{dom}(f)$. (The case $x_{0} \geqslant x$ for all $x \in \operatorname{dom}(f)$ can be treated analogously.) Suppose for simplicity that $x_{0}=0$ and $f\left(x_{0}\right)=0$ and consider a sequence $\left(x_{n}\right)_{n}$ in $\operatorname{dom}(f)$ such that $\lim _{n \rightarrow+\infty} x_{n}=0$. Since $f$ is lsc, one gets $\liminf _{n \rightarrow+\infty} f\left(x_{n}\right) \geqslant 0$. Suppose now that there exists $x$ in $\operatorname{dom}(f)$ with $f(x)>0$. From convexity of $f$ it follows that $f(t x) \leqslant t f(x)$, for all $t \in[0,1]$, hence in particular $\lim _{t 0_{0^{+}}} f(t x)=0$. It follows easily that $\limsup _{n \rightarrow+\infty} f\left(x_{n}\right) \leqslant 0$.

The above result fails in $R^{2}$ even if $T$ is maximal cyclically monotone. This is shown by the following example:

EXAMPLE. Consider the function $f: R^{2} \rightarrow R \cup\{+\infty\}$ given by the formula

$$
f\left(x_{1}, x_{2}\right)= \begin{cases}x_{2}^{2} / x_{1} & \text { if } x_{1}>0 \\ 0 & \text { if } x_{1}=x_{2}=0 \\ +\infty & \text { elsewhere }\end{cases}
$$

In $[4, \mathrm{p} .83]$ it has been shown that $f$ is convex and lower semicontinuous. This function is a classical example of a non asymptotically well behaved function and has been used several times in the past. We show that the operator $T=\partial f$ is not $\sigma$-cyclically monotone. Note first that $f$ is differentiable at every non-zero point of its domain, with gradient $\nabla f\left(x_{1}, x_{2}\right)=\left(-\left(x_{2} / x_{1}\right)^{2}, 2\left(x_{2} / x_{1}\right)\right)$. Set now $x_{n}=\left((1 /(n+1))^{3}, 1 /(n+1)\right)$ and $x_{n}^{*} \in$ $\partial f\left(x_{n}\right)=\left\{\nabla f\left(x_{n}\right)\right\}$. It follows that $\lim _{n \rightarrow+\infty} x_{n}=0$ and that $x_{n}^{*}\left(x_{n+1}-x_{n}\right)>0$, for $n \geqslant 1$. Since $f$ attains its minimum at $x_{0}=0$, it follows that $0 \in \partial f\left(x_{0}\right)$. Setting $x_{0}^{*}=0$ we obtain:

$$
\limsup _{n \rightarrow \infty}\left\{x_{0}^{*}\left(x_{1}-x_{0}\right)+\sum_{i=1}^{n} x_{i}^{*}\left(x_{i+1}-x_{i}\right)\right\}>0
$$

which shows that $T$ is not $\sigma$-cyclically monotone.

One may observe that in the previous example the domain of $T$ is included in a half-space. This condition is in fact indispensable for such examples in finite dimensional spaces. This is shown by the following proposition. 
Proposition 6. Let $T: R^{n} \rightarrow 2^{R^{n}}$ be a densely defined cyclically monotone operator. Then $T$ is $\sigma$-cyclically monotone.

Proof: Since $T$ is cyclically monotone, we conclude from Theorem 2 that for some convex lsc function $f$ we have $T \subseteq \partial f$. Since $\operatorname{dom}(T)$ is dense in $R^{n}$ and $\operatorname{dom}(T) \subseteq$ $\operatorname{dom}(\partial f) \subseteq \operatorname{dom}(f)$, it follows that $\operatorname{dom}(f)$ is also dense. Since the latter set is convex, we infer that $\operatorname{dom}(f)=R^{n}$, hence $f$ is continuous. The result now follows from Proposition 4.

The statement of Proposition 6 is no longer true in infinite dimensional spaces, even if one assumes $T$ to be maximal (that is, equal to the subdifferential of a lsc convex function $f$ ). We shall use the following result, which is a version of the approximate mean value theorem for the Clarke-Rockafellar subdifferential, see [1, Corollary 4.3]:

Proposition 7. Let $f: X \rightarrow R \cup\{+\infty\}$ be a lsc function and $a, b \in X$ be such that $f(a)<f(b)$. Then there exist $c \in[a, b], c \neq b$, and sequences $\left(x_{n}\right)_{n \in N}$ in $X$ and $\left(x_{n}^{*}\right)_{n \in N}$ in $X^{*}$ with $x_{n}^{*} \in \partial^{\dagger} f\left(x_{n}\right), x_{n} \rightarrow c, f\left(x_{n}\right) \rightarrow f(c)$ such that:

$$
x_{n}^{*}\left(x-x_{n}\right)>0
$$

for all $n \in N$ and every $x=c+t(b-a)$, with $t>0$.

Proposition 8. There exists a lsc convex function $f$ on $\ell^{2}(N)$ such that its subdifferential $\partial f$ is densely defined without being $\sigma$-cyclically monotone.

Proof: Let $X=\ell^{2}(N)$. We consider the function $f: X \rightarrow R \cup\{+\infty\}$ defined for every $x=\left(x_{i}\right)_{i=1}^{+\infty} \in X$ as follows:

$$
f(x)=\|x\|_{1}:=\sum_{i=1}^{+\infty}\left|x_{i}\right| .
$$

Since $f$ is the pointwise supremum of the convex continuous functions $\varphi_{n}: X \rightarrow R$ given by $\varphi_{n}(x)=\sum_{i=1}^{n}\left|x_{i}\right|$, it follows that $f$ is lower semicontinuous and convex. From [5] (or [3]) we have that $\partial f: X \rightarrow 2^{X^{\bullet}}$ is maximal cyclically monotone. We shall show that $\partial f$ is not $\sigma$-cyclically monotone.

Let us first observe that $\operatorname{dom}(f)=\ell^{1}(N)$. On the other hand one can easily see that $f$ is nowhere continuous. Using the inclusions $\ell^{1}(N) \subset \ell^{2}(N) \subset \ell^{\infty}(N):=\left[\ell^{1}(N)\right]^{*}$, the relation $\left[\ell^{2}(N)\right]^{*}=\ell^{2}(N)$ and the fact that $f(y)=+\infty$ for all $y \in \ell^{2}(N) \backslash \ell^{1}(N)$, it follows from (1) that for every $x \in \operatorname{dom}(f)$ :

$$
\partial f(x)=\partial\left(\|\cdot\|_{1}\right)(x) \cap \ell^{2}(N)
$$

where $\partial\left(\|\cdot\|_{1}\right)(x)$ denotes the usual subdifferential of the norm $\|\cdot\|_{1}$ in $\ell^{1}(N)$, as a subset of $\ell^{\infty}(N)$. 
Relation (8) shows that $\operatorname{dom}(\partial f)$ is dense in $X$, since it contains for instance the space $c_{00}(N)$. Setting now $x=0$ in (8) we obtain in particular that:

$$
\partial f(0)=\left\{y=\left(y_{n}\right)_{n} \in \ell^{2}(N):\left|y_{n}\right| \leqslant 1\right\}
$$

We shall need the following claim.

Claim. There exists a sequence $\left(y_{n}\right)_{n=1}^{+\infty}$ in $\operatorname{dom}(f)$ such that for all $n \geqslant 1$ :

(i) $y_{n} \in B_{1 / n}(0)$

(ii) $f\left(y_{n}\right)<f\left(y_{n+1}\right)$

(iii) the restriction of $f$ to the segment $\left[y_{n}, y_{n+1}\right]$ is strictly increasing.

Proof: We first note that for every $\varepsilon>0$, the function $f$ takes arbitrarily large finite values in the ball $B_{\varepsilon}\left(x_{0}\right)$.

Set now $k_{1}=1$ and $y_{1}=e_{1}:=(1,0,0, \ldots)$. For $\alpha_{2} \in R$ and $k_{2} \geqslant k_{1}+1$ we consider:

$$
y_{2}=\sum_{i=k_{1}+1}^{k_{2}} a_{2} e_{i}:=\left(0, a_{2}, a_{2}, \ldots, a_{2}, 0,0, \ldots\right)
$$

Imposing $\left\|y_{2}\right\|=1 / 2$ and $f\left(y_{2}\right)=2$ we conclude that $a_{2}=1 / 8$ and $k_{2}-1=16$. One can also check directly that condition (iii) is also satisfied for the segment $\left[y_{1}, y_{2}\right]$.

We shall now use induction to construct a sequence $\left(y_{n}\right)_{n=1}^{+\infty}$ in $c_{00}(N)$, such that for every $n \geqslant 1,\left\|y_{n}\right\|=1 / n, f\left(y_{n}\right)=n$ and the restriction of the function $f$ to the segment $\left[y_{n-1}, y_{n}\right]$ is strictly increasing. To this end, suppose that for $n \geqq 1$ we have defined $y_{n} \in c_{00}(N), k_{n} \in N$ and $\alpha_{n} \in R$ such that

$$
y_{n}=\sum_{i=k_{n-1}+1}^{k_{n}} \alpha_{n} e_{i}:=\left(0,0, \ldots, 0, \alpha_{n}, \alpha_{n}, \ldots, \alpha_{n}, 0,0, \ldots\right)
$$

where $\left(k_{n}-k_{n-1}\right) \alpha_{n}=n$ and $\left(k_{n}-k_{n-1}\right) \alpha_{n}^{2}=1 / n^{2}$. We then define $($ for $n+1)$

$$
y_{n+1}=\sum_{i=k_{n}+1}^{k_{n+1}} \alpha_{n+1} e_{i}:=\left(0,0, \ldots, 0,0, \ldots, 0, \alpha_{n+1}, \alpha_{n+1}, \ldots, \alpha_{n+1}, 0,0, \ldots\right)
$$

where $k_{n+1}$ and $\alpha_{n+1}$ are such that $\left(k_{n+1}-k_{n}\right) \alpha_{n+1}=n+1$ and $\left(k_{n+1}-k_{n}\right) \alpha_{n+1}^{2}=$ $1 /(n+1)^{2}$. It now suffices to check that the function

$$
t \rightarrow \rho(t)=f\left(y_{n}+t\left(y_{n+1}-y_{n}\right)\right)
$$

is increasing, for $t \in[0,1]$. Indeed, using (7), (11) and (12), a direct computation gives:

$$
\rho(t)=(1-t)\left(k_{n}-k_{n-1}\right) \alpha_{n}+t\left(k_{n+1}-k_{n}\right) \alpha_{n+1} \text {. }
$$

It follows that $\rho^{\prime}(t)=\left(k_{n+1}-k_{n}\right) \alpha_{n+1}-\left(k_{n}-k_{n-1}\right) \alpha_{n}=(n+1)-n=1>0$. The proof of the claim is complete.

We now construct a sequence $\left(x_{n}\right)_{n=0}^{+\infty} \subset \operatorname{dom}(\partial f)$ such that: 
(a) $\left\|x_{n}-y_{n}\right\| \leqslant\left\|y_{n}\right\|, n=1,2, \ldots$

(b) $x_{n}^{*}\left(x_{n+1}-x_{n}\right)>0$, for all $n \in N$.

Let $x_{0}=0$. Using (9) we can find some $x_{0}^{*} \in \partial f(0)$ such that $x_{0}^{*}\left(y_{1}-x_{0}\right)>0$. Consider now the open set $U_{0}=\left\{x^{\prime} \in X: x_{0}^{*}\left(x^{\prime}-x_{0}\right)>0\right\}$. Take any $z_{2} \in U_{0} \cap\left[y_{1}, y_{2}\right] \cap B_{\left\|y_{1}\right\|}\left(y_{1}\right)$. By the condition (iii) of the above claim we have $f\left(z_{2}\right)>f\left(y_{1}\right)$. Since the function $f$ is convex and lower semicontinuous, its convex subdifferential $\partial f$ coincides with the ClarkeRockafellar subdifferential $\partial^{\dagger} f$. Hence, since $y_{2}=y_{1}+\lambda\left(z_{2}-y_{1}\right)$ for some $\lambda>1$, applying Proposition 7 (for $a=y_{1}$ and $b=z_{2}$ ) we conclude the existence of some $x_{1} \in B_{\left\|y_{1}\right\|}\left(y_{1}\right) \cap U_{0}$ and some $x_{1}^{*} \in \partial f\left(x_{1}\right)$ such that $x_{1}^{*}\left(y_{2}-x_{1}\right)>0$. Note also that since $x_{1} \in U_{0}$ we have $x_{0}^{*}\left(x_{1}-x_{0}\right)>0$.

We further set $U_{1}=\left\{x^{\prime} \in X: x_{1}^{*}\left(x^{\prime}-x_{1}\right)>0\right\}$. Since $U_{1} \cap\left[y_{2}, y_{3}\right] \cap B_{\left\|y_{2}\right\|}\left(y_{2}\right)$ is nonempty, we may choose $z_{3} \in U_{1} \cap\left[y_{2}, y_{3}\right] \cap B_{\left\|y_{2}\right\|}\left(y_{2}\right)$. Condition (iii) again guarantees that $f\left(z_{3}\right)>f\left(y_{2}\right)$. Repeating the above arguments one inductively constructs a sequence $\left(x_{n}\right)_{n=0}^{+\infty}$ having properties (a) and (b).

Since $\left\|y_{n}\right\| \rightarrow 0$, it follows from (a) that $\lim _{n \rightarrow \infty} x_{n}=0:=x_{0}$. It follows now directly from (b) that $\partial f$ is not $\sigma$-cyclically monotone.

The above counterexample shows that, in infinite dimensional spaces, maximal cyclically monotone operators (having even a dense domain) may fail to be $\sigma$-cyclically monotone. However this can not happen if the operator $T$ is locally bounded on its domain. More generally we say that an operator $T$ has a locally bounded selection on a subset $D$ of its domain, if for every $x_{0} \in X$ there exists $M>0$ and $\delta>0$ such that:

$$
\forall z \in D \cap B_{\delta}\left(x_{0}\right), \exists z^{*} \in T(z):\left\|z^{*}\right\| \leqslant M
$$

We are now ready to state the following result for infinite dimensional spaces.

Propos ITION 9. Let $T$ be a densely defined cyclically monotone operator that admits a locally bounded selection on its domain. Then $T$ is $\sigma$-cyclically monotone.

Proof: Using Theorem 2 we conclude that $T \subseteq \partial f$, for some convex lsc function $f$. It follows that $\partial f$ has a locally bounded selection on $\operatorname{dom}(T)(\subseteq \operatorname{dom} \partial f)$. From Proposition 4 it suffices to show that $f$ is continuous. To this end, consider any $x_{0} \in X$ and choose $M>0$ and $\delta>0$ so that (13) is satisfied. Since $\operatorname{dom}(T)$ is dense on $X$, there exists a sequence $\left(x_{n}\right)_{n \in N}$ in $\operatorname{dom}(\partial f) \cap B_{\delta}\left(x_{0}\right)$ such that $\lim _{n \rightarrow \infty} x_{n}=x_{0}$ and $x_{n}^{*} \in \partial f\left(x_{n}\right)$, with $\left\|x_{n}^{*}\right\| \leqslant M$. Take now any $x \in B_{\delta}\left(x_{0}\right) \cap \operatorname{dom}(f) \neq \emptyset$. It follows from (1) that $f\left(x_{n}\right) \leqslant f(x)+x_{n}^{*}\left(x_{n}-x\right)$. Since $f$ is lower semicontinuous, taking the limit as $n \rightarrow+\infty$ we conclude

$$
f\left(x_{0}\right) \leqslant f(x)+M\left\|x_{0}-x\right\|
$$

It follows that $x_{0} \in \operatorname{dom}(f)$, hence $\operatorname{dom}(f)=X$ and $f$ is continuous. 
Final CONClusion. Most arguments used in the proof of Proposition 8 are also valid for subdifferentials of lsc convex functions $f$ for which the restriction $f_{\text {(dom }}(\partial f)$ has a discontinuity at some $x_{0} \in \operatorname{dom}(\partial f)$. Consequently one expects that a subdifferential $\partial f$ of a lsc convex function $f$ is $\sigma$-cyclically monotone (if and) only if $f_{\text {ldom }(\partial f)}$ is continuous. This conjecture would be positively answered if for example one could establish the validity of the claim in the proof of Proposition 8 for the general case.

\section{REFERENCES}

[1] D. Aussel, J.-N. Corvellec and M. Lassonde, 'Mean value property and subdifferential criteria for lower semicontinuous functions', Trans. Amer. Math. Soc. 347 (1995), $4147-4161$.

[2] F.H. Clarke, Optimization and nonsmooth analysis (Wiley Interscience, New York, NY, 1983).

[3] R. Phelps, Convex functions, monotone operators and differentiability (2nd edition), Lecture Notes in Mathematics 1364 (Springer-Verlag, Berlin, 1991).

[4] R.T. Rockafellar, Convex analysis (Princeton University Press, Princeton NJ, 1970).

[5] R.T. Rockafellar, 'On the maximal monotonicity of subdifferential mappings', Pacific $J$. Math. 33 (1970), 209-216.

[6] R.T. Rockafellar, 'Generalized directional derivatives and subgradients of nonconvex functions', Canad. J. Math. 32 (1980), 257-280.

Laboratoire de Mathématiques Appliquées

CNRS UPRES A 5033

Université de Pau et des Pays de l'Adour

avenue de l'Université

$64000 \mathrm{Pau}$

France

e-mail: aris.daniilidis@univ-pau.fr 